\title{
Zur Einleitung: Grenzziehungen der Solidarität
}

\author{
Annette Schnabel • Ulf Tranow
}

Online publiziert: 28 . Oktober 2020

(C) Der/die Autor(en) 2020

Zusammenfassung Solidaritäten sind von beschränkter Reichweite und gehen mit Grenzziehungen einher. Diese sind historisch kontingent und gesellschaftlich umstritten. Eine Vielzahl gesellschaftlicher Konflikte lassen sich soziologisch als Solidaritätskonflikte deuten, in denen widerstreitende Ideen über Zugehörigkeit, Zusammengehörigkeit und wechselseitige Verantwortung verhandelt werden. Ausgehend von der Vorstellung, dass Solidarnormen den Kern des soziologischen Solidaritätsverständnisses ausmachen, geht der Beitrag der Frage nach, was zentrale Dimensionen der Grenzziehung sind. Es wird vorgeschlagen, zwischen sozialen und substanziellen Grenzziehungen zu unterscheiden. Soziale Grenzziehungen beziehen sich darauf, welche Personen(gruppen), Kollektive und Spezies (nicht) zu einem solidarischen „Wir“ bzw. zum Solidaritätsradius gezählt werden. Durch soziale Grenzziehungen wird somit bestimmt, wer (keine) Chancen auf Zuwendung, Aufmerksamkeit und Unterstützung hat. Substanzielle Grenzziehungen adressieren dagegen, welche materiellen oder immateriellen Solidarleistungen die Mitglieder eines Solidaritätsradius in welcher Höhe einander schulden und welchen Verbindlichkeitsgrad Solidarität hat. Durch substanzielle Grenzziehungen bestimmen sich damit Anspruchsniveau und Stärke von Solidarität. In beiden Dimensionen sind Grenzziehungen gekoppelt an legitimierende Ideologien und sozialstrukturelle Voraussetzungen. Ein soziologisches Verständnis empirisch beobachtbarer Solidaritätsverhältnisse verlangt, dass das Zusammenspiel beider Dimensionen rekonstruiert wird und die Mechanismen offengelegt werden, auf denen Grenzziehungen in beiden Dimensionen basieren.

\footnotetext{
A. Schnabel $(\bowtie) \cdot U$. Tranow

Institut für Sozialwissenschaften, Abteilung Soziologie, Heinrich-Heine-Universität Düsseldorf, Universitätsstr. 1, 40225 Düsseldorf, Deutschland

E-Mail: schnabel@hhu.de

U. Tranow

E-Mail: tranow@hhu.de
} 
Schlüsselwörter Solidarität · Solidaritätsradius · Grenzziehungen · Normen · Identität · Kooperation · Verantwortung

\title{
By way of introduction: Demarcations of solidarity
}

\begin{abstract}
Solidarities are of limited scope and are accompanied by demarcations. The latter are historically contingent and socially contested. Many social conflicts can, sociologically, be interpreted as solidarity conflicts in which opposing ideas about belonging, togetherness and mutual responsibility are being negotiated. Based on the idea that solidarity norms are at the core of the sociological understanding of solidarity, the paper asks about the central dimensions of demarcation. It is suggested to distinguish between social and substantial demarcations. Social demarcations refer to the persons, collectives and species that are (not) constituting the solidary "we" or part of the radius of solidarity. Social demarcations thus determine who gets a chance (or not) at attention, consideration and support. Substantial demarcations, on the other hand, address the material or immaterial benefits that the members of a radius of solidarity owe to each other and to what extent solidarity is obligatory. Substantial demarcations thus determine the aspiration level and strength of solidarity. In both dimensions, demarcations are linked to legitimizing ideologies and socio-structural preconditions. A sociological understanding of empirically observable relations of solidarity requires that the interplay of both dimensions be reconstructed and the mechanisms on which demarcations in both dimensions are based be revealed.
\end{abstract}

Keywords Solidarity · Radius of solidarity · Demarcation · Norms · Identity · Cooperation · Responsibility

\section{Introduction: la démarcation des frontières de la solidarité}

Résumé Les solidarités ont une portée limitée et s'accompagnent de la démarcation de frontières. Ces dernières sont historiquement contingentes et socialement controversées. Une multitude de conflits sociaux peuvent être interprétés sociologiquement comme des conflits de solidarité opposant des conceptions divergentes de l'appartenance, de la cohésion collective et de la responsabilité. En partant de l'idée que les normes de solidarité constituent le noyau de la conception sociologique de la solidarité, l'article s'interroge sur les principales dimensions de ces démarcations. Il est proposé d'établir une distinction entre frontières sociales et frontières substantielles. La démarcation des frontières sociales fait référence à la question de savoir quels (groupes de) personnes, collectifs et espèces (ne) font (pas) partie d'un «nous» solidaire ou d'un périmètre de solidarité. La démarcation des frontières sociales détermine qui (n') a (pas) une chance de recevoir de l'attention, de l'aide et du soutien. La démarcation des frontières substantielles concerne en revanche la nature et l'étendue des prestations de solidarité matérielles ou immatérielles que les membres d'un périmètre de solidarité se doivent mutuellement ainsi que le degré d'obligation attaché à la solidarité. La démarcation des frontières substantielles détermine le niveau d'exigence et la force de la solidarité. Dans ces deux dimensions, les démarcations sont associées à des idéologies de légitimation et à des conditions 
sociostructurelles. Une conception sociologique des rapports de solidarité empiriquement observables exige de reconstruire l'interaction entre ces deux dimensions et de mettre au jour les mécanismes sur lesquels est basée la démarcation des frontières dans ces deux dimensions.

Mots-clés Solidarité · Démarcation de frontières · Normes · Identité · Coopération · Responsabilité

\section{Einleitung}

Die aktuellen gesellschaftlichen Debatten über die Klimakrise, den Sozialstaat, die Migrationsfrage oder die Verfasstheit der EU lassen sich als Solidaritätsdiskurse interpretieren, in denen konfligierende Vorstellungen über Zugehörigkeit, Zusammengehörigkeit und kollektive Verantwortung verhandelt werden. Die Positionen in diesen Konfliktfeldern unterscheiden sich darin, wer zum solidarischen „Wir“ gezählt wird und welche Leistungen sich die Mitglieder einer Solidargemeinschaft schulden. Solidaritäten sind immer von beschränkter Reichweite und deswegen untrennbar mit Fragen der Grenzziehung verknüpft. Durch Grenzziehungen wird bestimmt, welche Personen(gruppen) und Anliegen Chancen auf Aufmerksamkeit, Zuwendung und Unterstützung haben. Grenzziehungen der Solidarität sind historisch höchst variabel. Zum Gegenstand sozialer Auseinandersetzung werden sie immer dann, wenn die ihnen zugrundeliegenden Wir-Konzepte und damit die mit ihnen verbundenen Ethiken und Machtkonstellationen ihre Selbstverständlichkeit verlieren. Grenzziehungen der Solidarität sind gekoppelt an Ideologien, durch die normative Konstruktionen von Gemeinschaftlichkeit und Verantwortung angeleitet werden. Durkheim (1992 [1893]) verdanken wir die soziologische Einsicht, dass Grenzziehungen nicht auf frei flottierende Ideologien reduziert werden können, sondern in sozialstrukturelle Voraussetzungen eingebettet bleiben. Starke Solidarverpflichtungen bei gleichzeitig strikten Grenzziehungen plausibilisieren und institutionalisieren sich am ehesten unter der Bedingung geringer interner als signifikant anerkannter Differenzierung und hoher sozialer Interaktionsdichte der Mitglieder. Unter der Voraussetzung sozialer Heterogenität von als wichtig erachteten (Personen-)Merkmalen und der Partizipation von Individuen an einer Vielzahl ,sozialer Kreise“ (Simmel) werden dagegen Solidaritätsformen wahrscheinlicher, die in ihren Grenzziehungen durchlässiger und insgesamt weniger umfänglich ausfallen.

Im vorliegenden Schwerpunktheft des Berliner Journals für Soziologie werden Grenzziehungsfragen aus unterschiedlichen Perspektiven und im Hinblick auf unterschiedliche Solidaritätskontexte (u.a. Familie, Wohlfahrtsstaat, Weltwirtschaft) thematisiert. An dieser Stelle werden wir in das Thema einleiten, indem wir das soziologische Verständnis von Solidarität rekonstruieren und Dimensionen der Grenzziehung differenzieren. 


\section{Solidarität: Soziologische Konturen}

Solidarität ist ein schillernder Begriff. In alltäglichen wie politischen Diskursen wird er für alle möglichen Sachverhalte verwendet, die etwas mit Hilfe und kooperativem Zusammenhalt zu tun haben. Der Begriff ruft Assoziationen hervor, die von nachbarschaftlicher Unterstützung über ehrenamtliche Hilfe zugunsten Benachteiligter bis zu Arbeitskampf und Sozialstaat reichen (Dallinger 2009, S. 21). Als politischethischer Leitbegriff ist Solidarität seit Mitte des 19. Jahrhunderts etabliert; als soziologischer Fachterminus mit primär deskriptiv-analytischer Ausrichtung wurde er wesentlich durch Emile Durkheim (1992 [1893]) geprägt. Durkheims Unterscheidung zwischen einer auf Kollektivbewusstsein basierenden mechanischen und einer auf Interdependenz gründenden organischen Solidarität dürfte bis heute zu den populärsten Theoriefiguren der Soziologie gehören. Trotz der zentralen Bedeutung, die der Solidaritätsbegriff für die Entwicklung soziologischen Denkens gespielt hat, ist sein Stellenwert im Fach umstritten (Thome 1998). Während manche ihn für einen Schlüsselbegriff halten und Solidarität zum ,fundamental point for sociological analysis“ (Alexander 1980, S. 6) erklären, bezweifeln andere seinen analytischen Wert. Niklas Luhmann (1984) etwa erklärt Solidarität zu einem soziologisch wenig gehaltvollen Begriff, dessen weite Verbreitung sich eher durch seinen Grundwertcharakter als durch seine analytische Substanz erklären lasse.

Tatsächlich ist kaum von der Hand zu weisen, dass sich die Soziologie schwertut, die analytischen Potenziale dieses Begriffs zu bestimmen. Verglichen mit Schlüsselkonzepten wie Sozialstruktur, Herrschaft oder Schicht gibt es nur wenige Versuche, Solidarität theoretisch-konzeptionell auszubuchstabieren und für soziologische Fragestellungen fruchtbar zu machen. ${ }^{1}$ Trotz vielerlei Unschärfen in seiner Verwendung lassen sich dennoch Konturen eines allgemeinen soziologischen Solidaritätsverständnisses identifizieren. Diese erschließen sich, wenn danach gefragt wird, mit welchen Problembezügen und welchen empirischen Phänomenen Solidarität assoziiert wird.

Die klassische Sozialtheorie in der Durkheim-Parsons-Tradition verknüpft den Begriff der Solidarität mit dem Problem sozialer Ordnungsbildung. Hintergrund ist die Hobbes'sche Fragestellung, wie soziale Ordnung unter der Voraussetzung eigeninteressierter Akteur*innen und antagonistischer Interessen möglich sei. Ausgehend von der Überzeugung, dass weder die Kräfte des Marktes noch autoritative Macht hinreichend soziale Ordnung stiften könnten, wird ein grundlegender Moralbedarf attestiert. Die Lösung des als „transhistorisch“ und „unentrinnbar“ (Wrong 1994, S. 36) angenommenen Ordnungskonflikts verlange, dass moralische Regeln wirksam würden, die den Menschen ,zu Konzessionen, zu Kompromissen, zur Berücksichtigung höherer Interessen als seiner eigenen“ (Durkheim 1992 [1893], S. 284) verpflichteten. In der Durkheim-Parsons-Tradition wird Solidarität mit dem Wirksamwerden dieser moralischen Regeln gleichgesetzt. Der Problembezug von Solidarität ist aus dieser Perspektive identisch mit dem der normativen Integration von Gesellschaft.

\footnotetext{
1 Beispiele für solche Versuche sind: Baum (1975), Kaufmann (1984), Hechter (1987), Thome (1998), Lindenberg (1998) und Tranow (2012).
} 
In der üblichen soziologischen Verwendungsweise des Begriffs dominiert dagegen ein engeres Problemverständnis. Solidarität wird nicht mit dem Problem normativer Integration insgesamt identifiziert, sondern mit spezifischen Herausforderungen der normativen Ordnung verknüpft. Analog zum Alltagsverständnis sind es Kooperationsherausforderungen wie die Trittbrettfahrerproblematik sowie Unterstützungsund Hilfsbedürftigkeiten, die den Problemhorizont von Solidarität definieren. Solidarität in diesem Sinne ist ein Querschnittsthema und wird in verschiedenen Bindestrich-Soziologien untersucht, von der Familiensoziologie (Huinink et al. 2001; Knijin und Komter 2004) über die Soziologie des Sozialstaats (Kaufmann 2002; Dallinger 2009) bis zur Soziologie internationaler Beziehungen (Harnisch et al. 2009). Der weite und enge Problembezug konvergieren darin, dass Solidarität mit der Frage verknüpft wird, wie sich Kollektive bilden und reproduzieren, in denen die Mitglieder partikulare Identitäten und Interessen zugunsten der Bindung an gemeinsame Ziele zurückstellen und wechselseitig Verantwortung übernehmen.

Hinsichtlich der Frage, worin sich Solidarität empirisch manifestiert, lassen sich zwei dominante soziologische Perspektiven unterscheiden: Aus akteurstheoretischer Perspektive wird Solidarität als ein Handlungstypus und aus strukturtheoretischer Perspektive als Institution aufgefasst (Berger 2004, S. 254 ff.; Tranow 2012, S. 35 ff.; Laitinen und Pessi 2014, S. 3). Was Solidarität als Handlungstypus auszeichnet, wird in der Literatur sehr unterschiedlich definiert. Allerdings lässt sich eine Art kleinster gemeinsamer Nenner ausmachen: Solidarisches Handeln wird in der Regel als ein Kooperations- oder Unterstützungshandeln verstanden, das durch Wertvorstellungen von Gemeinschaftlichkeit und/oder kollektiver Verantwortung motiviert ist (ähnlich Thome 1998, S. 219). Solidarisch sind Kooperations- oder Unterstützungsleistungen also dann, wenn eine egoistische Interessenverfolgung im Handlungsvollzug fehlt oder zumindest nicht dominant ist (Kaufmann 2002, S. 44). ${ }^{2}$

Aus strukturtheoretischer Perspektive wird Solidarität mit institutionalisierten Kooperations- und Unterstützungsleistungen identifiziert. Die Solidarität einer Gruppe oder Gemeinschaft drückt sich hier in formell oder informell festgelegten und durch Kontrollmechanismen abgesicherten Kooperations- und Unterstützungsleistungen aus. Auf der Ebene des Nationalstaats sind dies bspw. die Steuersätze oder Sozialleistungen, auf der Ebene der Familie normativ abgesicherte Unterstützungspraktiken und Kollektivgutbeiträge wie die Care-Arbeit von Geschwistern gegenüber ihren bedürftigen Eltern. Auch institutionalisierte Solidarität weist einen Moralbezug auf. Dieser zeigt sich in Semantiken der Gemeinschaftlichkeit und kollektiven Verantwortung, durch die entsprechende Leistungen begründet oder eingefordert werden. Damit ist nicht ausgeschlossen, dass auch Eigeninteressen oder funktionale Erfordernisse in die Legitimation einfließen können, doch eine Bezugnahme auf Moral bleibt wesentlich, um institutionalisierte Leistungen als Ausdruck von Solidarität verstehen zu können.

Auch wenn akteurs- und strukturtheoretische Perspektiven an unterschiedlichen Enden des Sozialen ansetzen, weisen sie eine Konvergenz auf. Diese besteht darin,

\footnotetext{
2 Eine abweichende Vorstellung solidarischen Handelns findet sich bei Michael Hechter (1987). Aus Rational-Choice-Perspektive plädiert er dafür, solidarisches Handeln ausschließlich über erbrachte Leistungen zu bestimmen und von Motiven oder Emotionen als Definitionsmerkmalen abzusehen.
} 
dass jeweils Solidarnormen ins Zentrum eines soziologischen Solidaritätsverständnisses gesetzt werden. Während sich aus akteurstheoretischer Perspektive Solidarität in einer individuellen Bindung gegenüber diesen Solidarnormen ausdrückt, manifestiert sich Solidarität aus strukturtheoretischer Perspektive in ihrer Institutionalisierung.

Aus der Rekonstruktion der Problem- und Gegenstandsbezüge ergibt sich ein soziologisches Verständnis von Solidarität, welches den Begriff mit der normativen Konstruktion von Kooperations-, Unterstützungs- und Verantwortungsgemeinschaften assoziiert. Der normative Charakter von Solidarität besteht darin, dass ihr ein Anspruch des Sollens und Geboten-Seins zugrunde liegt, der sich aus der Mitgliedschaft zu einem „Wir-Kreis“ (Garcia 2018) ableitet. Der Verpflichtungs- und Institutionalisierungsgrad von Solidarnormen variiert und reicht von einer Selbstbindung aufgrund persönlicher Werthaltungen bis hin zur rechtlichen Verankerung bspw. im Rahmen des Wohlfahrtsstaates.

Soziologisch empfiehlt es sich, den Begriff der Solidarität nicht an eine bestimmte Ethik zu knüpfen, sondern ihn für unterschiedliche normative Ausdeutungen offen zu halten. Demnach handelt es sich etwa bei der Auseinandersetzung um die Migrationsfrage nicht um einen Konflikt, bei dem eine Position der Solidarität (,Flüchtlinge willkommen“) auf die einer Unsolidarität (,Grenzen schließen“) trifft, sondern um einen Clash von zwei Solidaritätsideologien. Die Vorstellung einer universellen menschenrechtlichen Solidarität steht hier im Widerstreit mit der Idee, dass die Solidarität einer (kulturell) integrierten nationalen Gemeinschaft Vorrang genieße (vgl. Ott 2016). Der Vorteil eines normativ offenen Solidaritätsbegriffs ist, dass sich mit ihm Solidarität als ein Konfliktgegenstand in unterschiedlichen sozialen Feldern verstehen lässt. In diesen konkurrieren divergierende und sich häufig ausschließende Kooperations-, Unterstützungs- und Verantwortungsnormen um gesellschaftliche Anerkennung und Institutionalisierung. Der wesentliche Unterschied und Konfliktpunkt zwischen konkurrierenden Solidaritätsideologien betrifft Fragen der Grenzziehung.

\section{Dimensionen der Grenzziehung von Solidarität}

Grenzziehungen scheiden ein Innen von einem Außen, konstruieren Differenzen und haben damit eine grundlegende Strukturierungsfunktion für soziale Gebilde. Dieses gilt auch für Solidarität: Familiale, politische, nationalstaatliche oder weltgesellschaftliche Solidaritäten konstituieren sich durch Grenzziehungen. Diese vollziehen sich in zwei zentralen Dimensionen, einer sozialen und einer substanziellen. Durch soziale Grenzziehungen wird bestimmt, welche Entitäten, also Personen, Gruppen, Kollektive oder Spezies zu einem ,Solidaritätsradius“"3 gehören sollen und damit Chancen auf Solidarität haben. Substanzielle Grenzziehungen beziehen sich dagegen auf Umfang und Anspruchsniveau von Solidarleistungen. Durch Grenzziehun-

\footnotetext{
3 Als ,,Solidaritätsradius“ lässt sich in Anlehnung an Markus Freitags (2001) „Vertrauensradius“ ein Kreis von Personen bezeichnen, innerhalb dessen Solidarnormen Geltung haben. Freitag schließt mit dem Begriff des Vertrauensradius an Francis Fukuyamas „radius of trust“ an (Fukuyama 1995, 2001).
} 
gen in diesen beiden Dimensionen bestimmt sich die Reichweite von Solidarität. Wesentlich ist, dass soziale und substanzielle Grenzziehungen in einem Interdependenzverhältnis stehen: Starke Solidaritäten institutionalisieren sich am ehesten in homogenen Wir-Kreisen mit dichten sozialen Beziehungen, während unter der Voraussetzung von Heterogenität und geringer Interaktionsdichte schwache, aber dafür inklusivere Solidarformen wahrscheinlicher werden. Homogenität und Heterogenität sind hierbei jedoch je nach Kontext sehr unterschiedlich bestimmt.

Wie oben bereits unter Rückgriff auf Durkheims Unterscheidung zwischen mechanischer und organischer Solidarität beschrieben, lassen sich zwei Grundlagen für Solidarität bestimmen: Zum einen kann Solidarität aus als signifikant erachteten Ähnlichkeiten erwachsen. Empirisch lassen sich solchermaßen begründete Solidaritätsverpflichtungen z. B. im Kontext religiöser Gemeinschaften oder ethnisch ausgewiesener Kollektive finden. Zum anderen kann sich Solidarität aber auch aus vertraglichen Verpflichtungen personenmerkmalsheterogener Personen und Personengruppen ergeben, wie sie z.B. in Formen , administrativer“ Solidaritäten zu finden sind (s. dazu auch den Beitrag von Annette Schnabel in diesem Heft).

\subsection{Soziale Grenzziehungen}

Der „Solidaritätsradius“ bestimmt die soziale Reichweite von Solidarität. In Anlehnung an Tristan Garcias (2018) Analyse von Wir-Strukturen lässt sich ein solcher Radius als ein auf Aus- und Zuschnitten basierendes „Einteilungsprinzip“ verstehen. Bei diesen Schnitten handelt es sich um Grenzziehungen, durch die sich ein Innen von einem Außen isoliert und das Innen in unterschiedlichen Präzisionsgraden differenziert wird. Soziale Grenzziehungen der Solidarität homogenisieren und heterogenisieren zugleich. Durch sie werden Personen(gruppen) auf die eine oder andere Seite einer Grenze gestellt, wobei im Hinblick auf die Binnenseite Geteiltheiten postuliert werden, die zu Solidarität verpflichten, und im Hinblick auf die Außenseite Differenzen behauptet werden, die von Solidarität entlasten.

Solidaritätsradien sind ausgesprochen variabel. Sie können sich auf höchst unterschiedliche Geltungsbereiche und Ebenen beziehen, auf kleinteilige Interaktionsgemeinschaften ebenso wie auf alle möglichen ,imagined communities“ (Anderson 1983). Analytisch lassen sich zwei Varianten des „Wir“ und mit ihnen verknüpfte Solidaritätsradien unterscheiden. Ein kommunitäres „Wir“ gründet auf erlebter Zusammengehörigkeit im Zuge einer geteilten Praxis kollektiver Identität. Der Radius eines kommunitären „Wir“ endet dort, wo eine kollektive Identitätspraxis ihre Grenzen findet. Solidarität kann diese Grenze allerdings auch überschreiten. Solidarität von Mitgliedern der Mehrheitsgesellschaft mit diskriminierten Minderheiten oder von Menschen im globalen Norden z. B. mit Opfern des Klimawandels im globalen Süden muss nicht auf erlebter Zusammengehörigkeit basieren, sondern kann sich alternativ auch auf Vorstellungen geteilter Zugehörigkeit beziehen. Die Wahrnehmung von geteilter Zugehörigkeit, bspw. zu einer räumlichen Einheit, zu einer politischen Entität, zur durch den Klimawandel bedrohten Weltgesellschaft oder zur Spezies der leidempfindsamen Wesen, kann die Vorstellung eines differenziellen „Wir“ begründen. Dieses kann Personen(gruppen) trotz unterschiedlicher Kollektividentitäten und 
divergenter Erfahrungshorizonte miteinander verbinden und zu Solidarität motivieren.

Zusammen- und Zugehörigkeiten leiten sich aus geteilten Merkmalen ab - erstere mit Bezug zu geteilten Kollektividentitäten und Erfahrungshorizonten, letztere mit Bezug auf geteilte Kategorien, die nicht notwendigerweise gleich identitäre Qualität entwickeln. Diese Merkmale transformieren sich allerdings nur dann zu einem „Wir“, wenn sie als moralisch signifikant erfahren und mobilisiert werden. ${ }^{4}$ Identitätstheoretische Ansätze in poststrukturalistischer Tradition informieren uns darüber, dass die Transformation von Merkmalen zu einem „Wir“ auf ein „konstitutives Außen“ und damit auf eine entschiedene Grenzziehung angewiesen ist (u.a. Derrida 1973; Triandafyllidou 1998; Lamont 2001). Das „Wir“ der Gläubigen konstituiert sich in Abgrenzung zu den Ungläubigen, das „Wir“ der Familie in Abgrenzung zu anderen Sozialverbänden ${ }^{5}$ und das „Wir“ des Hashtag-Aktivismus in Abgrenzung zu politischen Eliten. Aus dieser differenztheoretischen Überlegung folgt, dass erstens eine allumfassende Solidarität nur schwer vorstellbar ist und zweitens die Chance auf die Mobilisierung von Solidarität steigt, wenn sie sich auf eine signifikante und gesellschaftlich institutionalisierte Grenzziehung stützen kann. Richard Rorty knüpft an diese Überlegung an, wenn er die Verallgemeinerungsfähigkeit von Solidarität bestreitet. Er hält Solidaritätsgefühle dann für am stärksten, ,wenn die, mit denen wir uns solidarisch erklären, ,zu uns ' gehören und ,wir' etwas enger Begrenztes als die Menschenrasse ist" (Rorty 1992, S. 308). Bezogen auf die USA folgert er, dass die Mobilisierung von Solidarität zugunsten disprivilegierter afroamerikanischer Jugendlicher dann die größte Aussicht auf Erfolg hat, wenn sie als bedürftige amerikanische Mitbürger*innen statt lediglich als bedürftige Menschen erfahren werden. ${ }^{6}$

Aus dem kontrastiven Charakter von Solidarität leitet Lawrence Wilde ein ,pparadox at the heart of solidarity“ (Wilde 2007, S. 173) ab. Dieses sieht er darin, dass sich Zusammenhalt, Unterstützungsbereitschaft und wechselseitige Verantwortung dann am erfolgreichsten mobilisieren lassen, wenn das eigene „Wir“ in einem antagonistischen Verhältnis zu einem anderen wahrgenommen wird. Dieser Mechanismus einer „Solidaritätssteigerung durch Feindseligkeit“ (Prisching 2003) ist wesentlich, um Konfliktdynamiken erklären zu können. Diese sind zentral dadurch bestimmt, wie gut es Gruppen gelingt, mit Verweis auf die Feindseligkeit der jeweils anderen Partei intern Solidarität zu mobilisieren.

\footnotetext{
4 In diesem Zusammenhang sei auch an die Diskussion um die Kontakt-These (Allport 1954) und die Group-Threat-Theorie (Blumer 1958 und Blalock 1967) erinnert: Im Zusammenhang mit diesen beiden Theorien konnte verschiedentlich nachgewiesen werden, dass nicht alle Personenkategorien gleichermaBen für die Konstruktion von Gruppendifferenzen signifikant sind, sondern es bestimmter Voraussetzungen bedarf, damit potenzielle ,signifikante Andere“ zu tatsächlichen werden (u.a. Triandafyllidou 1998; Pettigrew 1998; Herjm 2007; Sigelman und Welch 1993).

5 Zu denken wäre hier etwa an Abgrenzungen des „Wir“ der Familie gegenüber dem Staat und seinen biopolitischen Eingriffsansprüchen.

6 Die empirische Mobilisierungskraft der „Wir“-Kategorie „Nation“ bedeutet allerdings nicht, dass sie eine quasi-natürliche Schwelle für die Ausdehnung des Solidaritätsradius darstellt. Die Mobilisierungskraft ist vielmehr ein Hinweis darauf, dass es sich bei der Nation um eine ,imagined community“ (Anderson 1983) handelt, die in den letzten 200 Jahren äußerst erfolgreich institutionalisiert und normalisiert wurde (vgl. Werron 2018).
} 
Auch wenn die negative Bezugnahme auf fremde Gruppen empirisch betrachtet einen wichtigen Mechanismus bei der Absteckung eines Solidaritätsradius darstellt, ist dieser nicht der einzige. Jodi Dean (1995) verweist darauf, dass Grenzziehungen der Solidarität auch auf gruppeninternen Abgrenzungen basieren können. Als Negativfolie kann hier bspw. die historische Verfasstheit der eigenen Gruppe fungieren, deren Solidarität im Lichte bestimmter Werte als defizitär beschrieben wird.

Ein Beispiel für einen solchen Mechanismus ist das selbstkritische Erinnern demokratischer Gesellschaften an die eigene menschenrechtsfeindliche und undemokratische Vergangenheit. Eine solche Erinnerungspolitik und solche kollektive Gedächtniskonstruktionen können zur normativen Selbstvergewisserung eines „Wir“ beitragen, die die Identität dieser Gesellschaften wesentlich bestimmt (Assmann 2018). So sieht Jan-Werner Müller (2010) in einer europäischen Erinnerungspolitik, bei der ein gemeinsamer Vorrat leidvoller Erfahrungen und selbstkritischer Geschichtsinterpretationen aufgebaut wird, einen Pfad zur Entwicklung einer verfassungspatriotischen europäischen Identität. Und tatsächlich lässt sich für die Mobilisierung der EU-Identität seit der Gründung der EU 1992 nicht nur ein räumliches, sondern auch ein zeitliches „Wir“ in Abgrenzung zu Nationalsozialismus und Kommunismus ausmachen (Alberth 2014). Allerdings weisen die aktuellen erinnerungspolitischen Debatten zum Holocaust in Deutschland und die Renaissance nationalistischer Rhetoriken in Europa darauf hin, dass selbstkritisches Erinnern ein höchst fragiler und voraussetzungsvoller Mechanismus der Wir-Konstitution zu sein scheint.

Will man die Grenzen von Solidaritätsradien verstehen, so ist eine Untersuchung der Mechanismen ihrer Konstruktion wesentlich. Die Frage nach sozialen Grenzziehungen ist damit soziologisch aber noch nicht erschöpfend behandelt. Da Individuen mehreren „Wir“ zugehörig sind und zudem solidarische Handlungsmotive mit anderen konkurrieren, kann nicht von bestimmten Zusammen- oder Zugehörigkeiten unumwunden auf ein entsprechendes solidarisches Handeln geschlossen werden. Verfügen Individuen bspw. über ein Klassenbewusstsein, folgt daraus nicht, dass sie sich in relevanten Situationen automatisch solidarisch im Sinne ihrer Klasse verhalten. Ein Klassenbewusstsein ist dafür zwar eine notwendige, doch keine hinreichende Bedingung: Die aktuellen politischen Konstellationen - z. B. in Frankreich, Großbritannien oder Deutschland - zeigen, dass sich Klassenzugehörigkeiten unter bestimmten Bedingungen durch ethnische oder religiöse Zugehörigkeiten und entsprechende Verpflichtungen zu Solidarität überschreiben lassen. ${ }^{7}$ Als eine Kollektividentität ist jedes „Wir“ zunächst nur ein Solidaritätspotenzial; damit Solidarität entsteht, muss es in entsprechenden Situationen und Kontexten aktualisiert werden. Aus handlungstheoretischer Sicht schließt sich an die Frage nach den Mechanismen der Wir-Konstruktion deswegen die Frage an, wie ein „Wir“ in spezifischen Situationen zur Anwendung kommt und die mit ihm verknüpften Solidaranforderungen für eine Situation deutungs- sowie handlungsleitend werden.

\footnotetext{
7 Zur exklusiven Verbindung von Klasse und Nationalismus in der Rassemblement National (ehem. Front National) vgl. Eribon (2016, S. 137), zur Liaison von Nationalismus und ,white working class“ im Brexit vgl. Evans (2017, S. 219).
} 
Einen möglichen Zugriff auf dieses Thema bieten handlungstheoretische Framing-Ansätze (Esser 2001; Lindenberg 2008). Aus dieser Perspektive lässt sich ein „Wir“ auf Individualebene als ein mentales Modell verstehen, das auf kollektive kognitive, ideologische und normative Wissensbestände zurückgreift und mit Handlungsskripten verknüpft ist. Mit der situationsspezifischen Aktualisierung eines bestimmten Wir-Frames erlangt dieses mentale Modell Dominanz für die subjektive Situationsdefinition. Die Beantwortung der Fragen, worum es in einer Situation geht, welche Ziele zu realisieren und welche Solidaransprüche dringlich sind, würde dann maßgeblich durch diesen Wir-Frame angeleitet. Andere Wir-Bezüge sowie individualistische Interessen sind dagegen in den Hintergrund verwiesen. $\mathrm{Ob}$ und in welcher Intensität Hintergrundidentitäten und -interessen Einfluss auf die Situationsdefinition nehmen können, wäre dann abhängig von der Salienz des dominanten Wir-Frames.

Um soziologisch erklären zu können, in welchen Situationen es wem gegenüber (nicht) zu Solidarität kommt, müssen somit Mechanismen der Aktualisierung von Wir-Frames berücksichtigt werden. Ein Kandidat für einen solchen Mechanismus sind diskursive Rahmungen. Wird eine gesellschaftliche Herausforderung bspw. nationalistisch gerahmt, provoziert dieses die Aktualisierung des nationalistischen Wir-Frames und verweist andere Identitäten und damit einhergehende Solidaritätsansprüche in den Hintergrund. Aus framingtheoretischer Perspektive ist zentral, dass sich aus der internen Rangordnung von Wir-Identitäten keineswegs eine stabile und transitive Ordnung von Solidaritätspräferenzen ableiten lässt. Die Praxis der Solidarität muss vielmehr als ein Effekt der Interaktion aus Wir-Identität und situationalen Faktoren ihrer Aktualisierung verstanden werden. Welche Personengruppen im Aufmerksamkeitsfokus stehen und solidarische Zuwendung erfahren, kann deswegen sehr volatil sein. Der Stimmungswechsel im Rahmen der sog. Flüchtlingskrise von 2015/16, bei der aus einer weit verbreiteten „Willkommenskultur“ heraus relativ abrupt an eine migrationsskeptische Haltung angeschlossen wurde, ist hierfür ein gutes Beispiel.

\subsection{Substanzielle Grenzziehungen}

Solidaritäten sind nicht nur in ihrer sozialen, sondern auch in ihrer substanziellen Reichweite beschränkt. Der substanzielle Kern von Solidarität besteht darin, dass zugunsten anderer oder einer Gemeinschaft bestimmte Leistungen erbracht werden. Diese Leistungen können unterschiedliche Bündel an Aufwendungen umfassen, die von Geld und anderen materiellen Leistungen bis hin zu Aufmerksamkeit, Anerkennung und Emotionsarbeit reichen. Inhalt und Umfang von erwarteten und erbrachten Solidarleistungen variieren sowohl zwischen als auch innerhalb von Wir-Kategorien. Im Rahmen der Familie oder dem Clan fallen Solidarleistungen in der Regel höher aus als innerhalb einer politischen Partei oder am Arbeitsplatz, wobei sich das Ausmaß innerfamilialer Solidarität zwischen Familien wiederum stark unterscheiden kann (vgl. Bühler-Niederberger in diesem Heft). Analog zur sozialen ist auch die substanzielle Grenzziehung von Solidarität umkämpft und Gegenstand von Aushandlungen. Im Kontext des Wohlfahrtsstaates drückt sich dies bspw. in den Auseinandersetzungen über die Art der Leistung (Zuwendungen ,in cash“ oder ,in 
kind") sowie über die Höhe der Leistungssätze für unterschiedliche Bedarfsgruppen aus; im Rahmen von Familien in Auseinandersetzungen darüber, wie viel Care-Arbeit Eltern ihren Kindern und Kinder wiederum ihren Eltern schulden und wie sich diese zwischen den Geschlechtern und unter den Kindern aufteilt.

Jene Regeln und Praxen, die die substanziellen Grenzen von Solidarität festlegen, bestimmen auch deren Anspruchsniveau bzw. deren Stärke. Michael Hechter (1987, S. 18) schlägt vor, die Stärke von Gruppensolidarität über den durchschnittlichen Anteil an Ressourcen zu bemessen, den Mitglieder einer Gruppe zugunsten kollektiver Ziele erbringen. Die Stärke der Solidarität von Gewerkschaften würde sich demnach darüber bestimmen, wie hoch der durchschnittliche Anteil an Zeit, Geld und anderen Ressourcen ausfällt, den die Mitglieder zur Erreichung ihrer kollektiven Ziele aufwenden. Hechters Vorschlag besticht durch formale Präzision und Empirienähe, doch er bleibt indifferent gegenüber der qualitativen Dimension von Solidarität. Diese gerät dagegen in den Blick, wenn bei der Bemessung der Stärke von Solidarnormen zusätzlich berücksichtigt wird, ob und wie sehr die durch sie verlangten Solidarleistungen von einem eigenen individuellen Nutzen aus der Solidarität entkoppelt sind (vgl. Baurmann 1998). Ein drittes Moment der Stärke von Solidarität ist ihr Verpflichtungscharakter, der von Freiwilligkeit bis hin zu rechtlich verfestigten Verpflichtungen reichen kann. Eine Rechtsverpflichtung zu bestimmten Solidarleistungen schließt freilich nicht aus, dass letztere verweigert werden können und dieses ggf. auch soziale Akzeptanz erfährt.

Jeder Solidaritätsradius impliziert, dass seine Mitglieder angemessene Beiträge zu Kollektivgütern leisten. Was als angemessen erscheint, wird vor dem Hintergrund unterschiedlicher Radien und der sie dominierenden Ideologien verschieden ausgelegt. Für die Bestimmung von Angemessenheit sind drei Prinzipien relevant, die je unterschiedlich realisiert werden: die Orientierung an individueller Leistungsfähigkeit, an Gleichheit sowie am individuellen Nutzen aus der Bereitstellung. Die drei Prinzipien unterscheiden sich darin, wie sehr individuelle Beitragsleistungen zu Kollektivgütern vom individuellen Nutzen ihrer Bereitstellung entkoppelt sind. Je stärker die Entkopplung, desto stärker der normative Solidaranspruch. Wird lediglich verlangt, dass ein Beitrag zum Kollektivgut geleistet wird, der dem Interesse der Beitragenden an der Bereitstellung entspricht, dann beschränkt sich der Solidaranspruch darauf, die anderen Mitglieder nicht auszubeuten. Wird von allen derselbe Beitrag verlangt, ist der Solidaranspruch höher, da in Bezug auf ein kollektives Anliegen individuelle Vorteile nicht verrechnet werden, sondern das Gleichheitsprinzip dominiert. Am stärksten sind Solidaransprüche dann, wenn die erwarteten Beitragsleistungen an der Leistungsfähigkeit bzw. Ressourcenausstattung orientiert sind.

Solidarität geht häufig mit Anreizen einher, Solidarleistungen zu umgehen oder über Gebühr in Anspruch zu nehmen. Anreize, Beiträge nicht zu leisten, liegen zum einen darin begründet, dass die aufzuwendenden (materiellen, zeitlichen, kognitiven, emotionalen) Ressourcen auch anderweitig für die Realisierung individueller, eigennütziger Interessen verwendet werden könnten: Statt den Eltern Weihnachten Gesellschaft zu leisten, lässt sich vielleicht konfliktfreier mit Freund*innen feiern; die hinterzogenen Steuern lassen sich vielleicht im Ausland individuell gewinnbringender anlegen; die Zeit, die für Organisation und Teilnahme an einer Soli- 
daritätskundgebung aufgewendet wird, lässt sich womöglich angenehmer im Kino verbringen. Zum anderen bestehen Anreize zur Zurückhaltung von Solidarleistungen darin, dass diese Leistungen Empfänger*innen außerhalb des von einem selbst präferierten Radius der Solidarität zugutekommen. Hier spielen also die Grenzziehungen und deren Anerkennung eine wichtige Rolle. Solidarleistungen können aber auch in ungerechtfertigt hohem Maße nachgefragt werden: Diskussionen um die vermeintliche Ausnutzung der Sozialsysteme machen das zum Thema.

Sowohl die Zurückhaltung als auch die übergebührliche Inanspruchnahme von Solidarleistungen unterliegt Sanktionen. Mit Heinrich Popitz (1980, S. 28) lassen sich Sanktionen als eine ,negativ (strafende) Antwort“ auf abweichende Handlungen verstehen, durch die das bekräftigt wird, wogegen verstoßen wurde. Sanktionen reichen hier von demonstrativer Missbilligung durch Teilöffentlichkeiten - z. B. beim „shaming and blaming“ abweichender Familienmitglieder - bis hin zu rechtlich verankerten Strafen durch eine Zentralinstanz. Sanktionshandlungen unterliegen ihrerseits ebenfalls einer Normierung, die sich u.a. auf den Grad der Verpflichtung zur Sanktionierung (darf oder muss sanktioniert werden?) und die Sanktionsschärfe bezieht (ebd., S. $48 \mathrm{ff}$.). Verpflichtungscharakter und Schärfe drohender Sanktionen verweisen auf die Verbindlichkeit von Solidarität, und diese lässt sich als ein weiterer Indikator für die Stärke bzw. Schwäche von Solidarnormen verstehen.

Wie kommt es nun zu substanziellen Grenzziehungen, d.h. wodurch wird bestimmt, wie hoch Solidarleistungen ausfallen, welche Form sie haben und welche Normen ihnen zugrunde liegen? Seit Durkheim (1992 [1893]) ist in der Soziologie die Denkfigur verankert, Art und Stärke von Solidarität an sozialstrukturelle Grundlagen zu koppeln. Durkheims These ist, dass sich im Zuge der arbeitsteiligen Differenzierung von Gesellschaft ein Wandel weg von einer lokal gebundenen und starken hin zu einer sozial erweiterten, dafür aber schwachen Solidarität vollzieht. Wie sozialstrukturelle Voraussetzungen und Solidaritätsstärke zusammenhängen, ist im Anschluss an Durkheim unterschiedlich ausbuchstabiert worden.

Randall Collins (2004) bietet eine Mikrofundierung von Durkheims These aus interaktionstheoretischer Perspektive. Dass sich Solidarität im Zuge einer arbeitsteiligen Differenzierung sozial erweitert und substanziell schwächt, erklärt sich mit Collins aus dem damit verbundenen Wandel der Interaktionsstrukturen. Die Hintergrundannahme ist, dass die Stärke von Solidarität mit der rituellen Intensität und sozialen Diversität von Interaktionen variiert. In der arbeitsteiligen Gesellschaft sind Individuen in eine Vielzahl loser und heterogener Netzwerke eingebunden, was zur Folge hat, dass Solidaritäten sich auf der einen Seite multiplizieren und auf der anderen Seite schwächer ausfallen, da die Interaktionsdichte innerhalb der Netzwerke vergleichsweise schwach ist. Mit Peter Berger (1970) ließe sich ähnlich argumentieren, dass in (westlich-)modernen Gesellschaften mit der Etablierung des Individuums als Adressat von Rechten und Pflichten und der sich etablierenden Dominanz des restitutiven Rechts eine De-Institutionalisierung von Gruppenzugehörigkeiten stattfindet. Die Konsequenz ist, dass Ehre als Modus der Vermittlung zwischen Individuum und Gesellschaft an Wichtigkeit verliert und durch den Modus der Würde ersetzt wird, der auch gegen Institutionen in Anschlag gebracht werden kann. Demzufolge ist es nicht mehr primär oder gar ausschließlich die Mitgliedschaft in einer exklusiven Gruppe, durch die sich Solidarität legitimiert, sondern durch die Umstel- 
lung von Ehre auf Würde genießen alle Personen qua ihres normativen Status als Individuen unbestreitbare und unveräußerliche Solidaransprüche. ${ }^{8} \mathrm{Ob}$ die hier beschriebene Entwicklung von einer zentrierten Solidarität hin zu einer diversifizierten Solidarität tatsächlich so eindeutig und unumkehrbar ist, wie diese Ansätze andeuten, mag allerdings bezweifelt werden. U.a. das Wiedererstarken nationalistischer Rhetoriken und das Wiederaufflammen von Ehre-Diskursen einerseits sowie die starken Solidarisierungen mit nicht-menschlichen Entitäten im Rahmen von Tierrechtsbewegungen andererseits erfordern eine stärker kontextualisierte Sicht auf starke und schwache Solidaritäten.

Ansätze in der Tradition der Rational-Choice-Theorie bieten eine alternative Perspektive auf den Zusammenhang von Sozialstruktur und Solidaritätsstärke. Ausgangspunkt ist die Vorstellung, dass sich Menschen zur Produktion und Konsumtion von ,,joint goods“ (Hechter 1987) in Solidargruppen zusammenschließen. Bei ,joint goods“ handelt es sich um Klubgüter, d. h. Nichtmitglieder haben keinen Zugang und Mitglieder können bei Fehlverhalten ausgeschlossen werden. Beispiele für ,joint goods“" sind Versicherungen, kollektive Produktionsmittel, geselliges Beisammensein oder ,welfare policies“. Die von einer Gruppe verlangten Beiträge zu ,joint goods“ werden von Hechter als „,membership tax“ (ebd., S. 41) verstanden. Die Frage nach der materiellen Grenzziehung übersetzt sich bei Hechter demzufolge in die Frage, wodurch sich die Höhe einer solchen Mitgliedschaftssteuer bestimmt. Neben spezifischen Eigenschaften der produzierten Güter sind Gruppengröße ${ }^{9}$ und vor allem Abhängigkeiten entscheidend. Je stärker Akteur*innen von einer Gruppe abhängig sind, um gewünschte Güter zu erhalten, desto größer können die Solidarverpflichtungen ausfallen. Ähnlich argumentiert Siegwart Lindenberg (1998), wenn er darauf hinweist, dass funktionale Interdependenz innerhalb von Gruppen entscheidend für ihre Solidaritätsstärke ist. Werden in einer Gruppe zentrale Güter geteilt, kann daraus eine Steigerungslogik der Solidarität folgen. Entschließt sich eine Gruppe von Farmer*innen, landwirtschaftliche Produktionsmittel, Schutz vor wilden Tieren und Kompensationsleistungen für Ernteausfälle als ,,joint goods“ bereitzustellen, dann ist die Wahrscheinlichkeit groß, dass daraus ein kollektives Interesse erwächst, zusätzliche Güter als kollektive Angelegenheit zu betrachten. Zu diesen kann Versorgung im Krankheitsfall zählen, da krankheitsbedingte Ausfälle das Ausbleiben von Beiträgen zu anderen ,,joint goods“ implizieren. Aber auch kollektive Geselligkeit kann dazu gehören, da durch sie soziale Kontrollmöglichkeiten

\footnotetext{
8 Die Umstellung von Ehre auf Würde wird von Berger wie folgt beschrieben: „The concept of honor implies that identity is essentially, or at least importantly, linked to institutional roles. The modern concept of dignity, by contrast, implies that identity is essentially independent of institutional roles. [...] In a world of dignity, the individual can only discover his true identity by emancipating himself from his socially imposed roles - the latter are only masks, entangling him in illusion, , alienation“ and ,bad faith “" (Berger 1970, S. 343).

9 Die Gruppengröße macht hier besonders dann einen Unterschied, wenn die zu erstellenden Güter einen hohen Fixkosten-Anteil haben, wenn sich also ein großer Anteil der Produktionskosten unabhängig von der Anzahl der Nutzenden auf die Beitragenden umlegen lässt. Steigen dagegen die Produktionskosten vielleicht sogar überproportional - mit der Anzahl der Nutzenden, wird es für die Beitragenden unattraktiv, die Nutzergruppe zu vergrößern. Dies gilt für die Ausbeutung natürlicher Ressourcen ebenso wie für die Grundrente oder die Emotionsarbeit in Freundeskreis und Familie.
} 
geboten werden. Verbunden ist dies jedoch meist mit der Solidaritätsverpflichtung, der so gebildeten Gemeinschaft gegenüber auch loyal zu sein.

\section{Perspektiven auf Solidarität}

Grenzziehungen in sozialer als auch in substanzieller Hinsicht sind konstitutiv für Solidarität: Ein soziologisches Verständnis des Gegenstands verlangt daher, dass nicht nur die inklusive und vergemeinschaftende Seite von Solidarität beobachtet wird, sondern auch die Grenzziehungen samt ihren Folgewirkungen, durch die wechselseitige Unterstützungs- und Verantwortungspraxen innerhalb eines Solidaritätsradius erst ermöglicht werden. Der vorliegende Schwerpunkt des Berliner Journals für Soziologie präsentiert Beiträge, die Grenzziehungen in unterschiedlicher Art und Weise und in unterschiedlichen Kontexten in den Blick nehmen und auf diese Weise zu einem gehaltvollen analytischen Verständnis von Solidarität beitragen. Den Beiträgen ist gemeinsam, dass sie Solidarität als besonderen Problemfall moderner, heterogener Gesellschaften begreifen und deren Potenziale der Gemeinwohlorientierung ausloten. Dies führt unweigerlich zu den beiden Fragen, wer das Gemeinwesen bildet, um dessen Wohl es geht, und auf welche Weise sich dieses als Solidarzusammenhang (re-)produziert.

In seinem theoretischen Beitrag adressiert Thilo Fehmel den interdependenten Zusammenhang zwischen Solidarität und Konflikt. Er schlägt ein sequenzanalytisches Modell vor, welches beide Formen der Vergesellschaftung und ihr Verhältnis zueinander deskriptiv, relational und prozessual abbildet. Ausgangspunkt des Modells ist ein Verteilungskonflikt. Aus diesem folgt Solidarität, sofern es zu einem Lösungsarrangement kommt, welches auf einer reziproken Einstandsverpflichtung basiert und mit wechselseitigen Erwartungen von Begünstigungen einhergeht. Der Konflikt ist damit aber nicht ein für alle Mal überwunden, sondern er bleibt latent vorhanden. Bei zunehmender Unzufriedenheit mit der praktizierten Verteilungslösung kann es zu einem manifesten Konflikt kommen, entweder zu einem „Konflikt um Solidarität“" oder zu „Konflikt statt Solidarität“. Bei Ersterem geht es lediglich um die Neujustierung von Solidarnormen innerhalb eines einvernehmlich anerkannten Solidaritätsradius; der Konflikt führt zu einem normativen sozialen Wandel innerhalb einer in sich beständigen Gruppe und trägt zu ihrer Integration bei. Bei Letzterem wird der Solidaritätsradius dagegen angezweifelt, und die Integration einer Gruppe steht auf dem Spiel.

Der Beitrag von Doris Bühler-Niederberger zeigt auf der Basis verschiedener qualitativer Materialien, dass familiale Solidarität neben einem geschlechtlichen auch einem generational organisierten Solidaritätsradius unterliegt. In fast allen Gesellschaften existieren starke Normen, die Kinder verpflichten, sich im Erwachsenenalter um ihre Eltern zu kümmern. Der Beitrag verweist allerdings darauf, dass diese Solidarnormen je nach kulturellem Kontext anders strukturiert sind: Insbesondere in Hinblick auf die substanzielle Dimension lassen sich zwei Modelle unterscheiden. Während das - v. a. im Globalen Norden dominante - ,Independenz-Modell““ (wohlfahrts-)staatlich und durch Expert*innen abgesichert auf die ,gute Kindheit“ rekurriert, verpflichtet das vermehrt im Globalen Süden anzutreffende „Interdependenz- 
Modell“ traditionsgemäß und rechtlich gestützt auf die Tugend des ,,guten Kindes“ (,filial piety“). Rekonstruiert man die Ergebnisse anhand des hier vorgeschlagenen Analyserahmens, lassen sich gleichwohl gemeinsame Muster der Grenzziehungen erkennen. In beiden Modellen ist der Solidaritätsradius eindeutig auf den familialen Kontext begrenzt; zur Solidarität verpflichtet hier allein der Status als Familienmitglied. Hier wie dort zeigen sich ,gute“ und ,schlechte“ substanzielle Formen der Normerfüllung: Letztere führen bei Nichterfüllung zu einem Ausschluss aus dem Solidarzusammenhang. Und schließlich führen die unterschiedlichen Systeme familialer Solidarnormen beiderseits zu systematischen Benachteiligungen - vor allem der durch die generationale Ordnung bestimmten Frauen, die verpflichtet werden, ihre eigene Berufsorientierung und materielle und soziale Unabhängigkeit zugunsten der familialen Emotions- und Care-Arbeit aufzugeben.

Der Beitrag von Annette Schnabel nimmt Wohlfahrtsstaatlichkeit als administrative Form der Solidarität in den Blick und fragt danach, ob und wie diese von anderen Formen der Solidarität abhängig ist. Administrative Solidarität wird dabei als ein Solidaritätsmodus adressiert, der die Solidarnormen der Kollektivgutbeteiligung, Redistribution, Unterstützung und Loyalität in besonderer Form zur Anwendung und Durchsetzung bringt. Auf der Basis einer quantitativen Mehrebenenanalyse kommt die Untersuchung zu dem Ergebnis, dass dieser Modus auch in den modernen Wohlfahrtsstaaten Europas nicht ganz ohne partikulare Solidarität, insbesondere in Form religiöser oder nationaler Zugehörigkeiten zu haben ist. Die Radien administrativer und partikularer Solidaritäten überschneiden sich also - und die Legitimation wohlfahrtsstaatlicher Policies hängt nicht zuletzt davon ab, dass deren soziale und substanzielle Grenzziehungen durch partikulare Grenzziehungen stabilisiert werden. Administrative Solidarität ist an staatliche Grenzziehungen gekoppelt, deren Übertretung in verschiedener Hinsicht Aushandlungsbedarfe provoziert: So muss verhandelt werden, welche Nicht-Staatsbürger*innen in welcher Weise im Inland administrative Solidarität in Anspruch nehmen dürfen und/oder zu ihr beizutragen haben. Hier verbinden sich soziale und substanzielle mit territorialen und zeitlichen Grenzen: Wer die ersteren überschreitet, wird zur Ausnahme und provoziert Legitimations- und Regelungsbedarf; die letzteren bestimmen wiederum darüber, wie grundsätzlich dieser Regelungsbedarf ausfällt. Erst wenn der Fremde kommt, um zu bleiben (oder der Einheimische geht, um zu verschwinden), werden die administrativen sozialen und substanziellen Grenzen wirkungsvoll und nachhaltig überwunden.

Auch Stephan Lessenich widmet sich in seinem Beitrag einer Analyse des wohlfahrtsstaatlichen Solidararrangements und den zugrundeliegenden Grenzziehungen. Ausgangspunkt ist die These, dass der demokratisch-kapitalistische Wohlfahrtsstaat Externalisierungseffekte zeitigt, die aus der gesellschaftlich verankerten „Basiserzählung“ über ihn systematisch ausgeblendet werden. Ausgeblendet wird, dass die „Kompromissstruktur“ des industriekapitalistischen nationalen Wohlfahrtsstaates ,funktionsnotwendig“ zulasten Dritter gehe, die nicht in seinen Solidaritätsradius fallen. Innerhalb der nationalen Wohlfahrtsstaaten sind dies Frauen in der Rolle von Reproduktionsarbeiterinnen und zugewanderte Erwerbstätige; jenseits der Wohlfahrtsstaatsgrenzen handelt es sich um all jene, die in ihren Lebenschancen durch die ökologischen und sozialen Verwerfungen eines globalisierten Kapitalismus beeinträchtigt werden, der die Basis des nationalen Wohlfahrtsstaates bildet. Anhand 
der sieben A's des Externalisierungskonzepts (Aneignung, Ausbeutung, Abwertung, Auslagerung, Abschließung, Ausblendung, Aufschiebung) wird dargelegt, dass genau jene Strukturmechanismen, die die Grundlage der materiellen (Re-)Produktion des europäischen Wohlfahrtsstaates darstellen, auch seine negativen Effekte auf Dritte begründen. Zwar breitet sich in den Zentren des demokratischen Wohlfahrtskapitalismus zunehmend eine ,,unheimliche Ahnung“ über die verdrängten Voraussetzungen und Folgewirkungen des wohlfahrtsstaatlichen Solidaritätsarrangements aus. Anstatt allerdings die Externalisierungseffekte als Solidaritätsproblem zu begreifen, sehe es derzeit eher so aus, als dass die Grenzziehungen eines exklusiven sozialpolitischen Zusammenhalts zunehmend offensiv und aggressiv verteidigt werden, etwa durch die symbolische Abwertung von Zugewanderten oder von Solidaransprüchen aus dem Globalen Süden.

Der Beitrag von Jörg Althammer und Maximilian Sommer schließlich widmet sich dem komplexen Verhältnis von Marktwirtschaft und Solidarität und fragt danach, wie marktförmige Inklusion und gemeinwohlorientierte Kooperation zusammenhängen. Solidarität zeigt sich hier auch in einer an Fairnessregeln gebundenen ,antagonistischen Kooperation“. Eine solche solidarisch-faire Kooperation ist jedoch daran gebunden, dass die Rahmenbedingungen für die Verhandlung bindender Verträge symmetrisch ausgestaltet werden, sodass (sozial) partikulare und (substanziell) individuell-nutzenmaximierende Interessen eingehegt werden. Symmetrische Verhandlungsbedingungen erlaubten dann, in einer globalisierten Welt die Verfolgung partikularer Interessen mit der kosmopolitischen Ambition einer gerechten Ordnung in Einklang zu bringen.

Funding Open Access funding enabled and organized by Projekt DEAL.

Open Access Dieser Artikel wird unter der Creative Commons Namensnennung 4.0 International Lizenz veröffentlicht, welche die Nutzung, Vervielfältigung, Bearbeitung, Verbreitung und Wiedergabe in jeglichem Medium und Format erlaubt, sofern Sie den/die ursprünglichen Autor(en) und die Quelle ordnungsgemäß nennen, einen Link zur Creative Commons Lizenz beifügen und angeben, ob Änderungen vorgenommen wurden.

Die in diesem Artikel enthaltenen Bilder und sonstiges Drittmaterial unterliegen ebenfalls der genannten Creative Commons Lizenz, sofern sich aus der Abbildungslegende nichts anderes ergibt. Sofern das betreffende Material nicht unter der genannten Creative Commons Lizenz steht und die betreffende Handlung nicht nach gesetzlichen Vorschriften erlaubt ist, ist für die oben aufgeführten Weiterverwendungen des Materials die Einwilligung des jeweiligen Rechteinhabers einzuholen.

Weitere Details zur Lizenz entnehmen Sie bitte der Lizenzinformation auf http://creativecommons.org/ licenses/by/4.0/deed.de.

\section{Literatur}

Alberth, L. (2014). Die Fabrikation europäischer Kultur. Zur diskursiven Sichtbarkeit von Herrschaft in Europa. Bielefeld: Transcript Verlag.

Alexander, J. C. (1980). Core solidarity, ethnic outgroup, and social differentiation: A multidimensional model of inclusion in modern societies. In J. Dofny \& A. Akiwowo (Hrsg.), National and ethnic movements (S. 5-28). Beverly Hills: Sage.

Allport, G. W. (1954). The nature of prejudice. Reading, MA: Addison-Wesley. 
Anderson, B. (1983). Imagined communities: Reflections on the origin and spread of nationalism. London: Verso.

Assman, A. (2018). Der lange Schatten der Vergangenheit. Erinnerungskultur und Geschichtspolitik. München: C. H. Beck.

Baum, R. C. (1975). The system of solidarities. A working paper in General Action Analysis. Indian Journal of Sociology, 16, 306-353.

Baurmann, M. (1998). Solidarität als soziale Norm und als Norm der Verfassung. In K. Bayertz (Hrsg.), Solidarität. Begriff und Problem (S. 345-388). Frankfurt a.M.: Suhrkamp.

Berger, J. (2004). Expandierende Märkte, schrumpfende Solidarität? Anmerkungen zu einer aktuellen Debatte. In J. Beckert, J. Eckert, M. Kohli \& W. Streeck (Hrsg.), Transnationale Solidarität. Chancen und Grenzen (S. 246-261). Frankfurt a.M.: Campus.

Berger, P. (1970). On the obsolescence of the concept of honour. Archives Européennes de Sociologie, 11, 339-347.

Blalock, H. M. (1967). Towards a theory of minority group relations. New York: Wiley.

Blumer, H. (1958). Race prejudice as a sense of group position. Pacific Sociological Review, 1, 3-7.

Collins, R. (2004). Interaction ritual chains. Princeton: Princeton University Press.

Dallinger, U. (2009). Die Solidarität der modernen Gesellschaft. Wiesbaden: VS Verlag für Sozialwissenschaften.

Dean, J. (1995). Reflective solidarity. Constellations, 2(1), 114-140.

Derrida, J. (1973). Speech and phenomena, and other essays on Husserl's theory of signs. Illinois: Northwestern University Press.

Durkheim, E. (1992 [1893]). Über soziale Arbeitsteilung. Studie über die Organisation höherer Gesellschaften. Frankfurt a.M.: Suhrkamp.

Eribon, D. (2016). Rückkehr nach Reims. Berlin: Suhrkamp.

Esser, H. (2001). Soziologie. Spezielle Grundlagen. Band 6: Sinn und Kultur. Frankfurt a.M.: Campus.

Evans, G. (2017). Brexit Britain: Why we are all postindustrial now. American Ethnologist, 44(2), 215-219.

Freitag, M. (2001). Das soziale Kapital in der Schweiz: vergleichende Einschätzungen zu Aspekten des Vertrauens und der sozialen Einbindung. Swiss Political Science Review, 7(4), 87-117.

Fukuyama, F. (1995). Trust: The social virtues and the creation of prosperity. New York: Free Press.

Fukuyama, F. (2001). Social capital, civil society and development. Third World Quarterly, 22(1), 7-20.

Garcia, T. (2018). Wir. Berlin: Suhrkamp.

Harnisch, S., Maull, H.W., \& Schieder, S. (Hrsg.). (2009). Solidarität und internationale Gemeinschaftsbildung, Beiträge zur Soziologie der internationalen Beziehungen. Frankfurt a.M.: Campus.

Hechter, M. (1987). Principles of group solidarity. Berkeley: University of California Press.

Hjerm, M. (2007). Do numbers really count? Group threat theory revisited. Journal of Ethnic and Migration Studies, 33(8), 1253-1275.

Huinink, J., Strohmeier, K.P., \& Wagner, M. (Hrsg.). (2001). Solidarität in Partnerschaft und Familie. Zum Stand soziologischer Theoriebildung. Würzburg: Ergon.

Kaufmann, F.-X. (1984). Solidarität als Steuerungsform - Erklärungsansätze bei Adam Smith. In F.-X. Kaufmann \& H.-G. Krüsselberg (Hrsg.), Markt, Staat und Solidarität bei Adam Smith (S. 158-185). Frankfurt a.M.: Campus.

Kaufmann, F.-X. (2002). Sozialpolitik zwischen Gemeinwohl und Solidarität. In H. Münkler \& K. Fischer (Hrsg.), Gemeinwohl und Gemeinsinn. Rhetoriken und Perspektiven sozial-moralischer Orientierung. Forschungsberichte der interdisziplinären Arbeitsgruppe „Gemeinwohl und Gemeinsinn“ der BerlinBrandenburgischen Akademie der Wissenschaften, Bd. 2 (S. 19-54). Berlin: Akademie Verlag.

Knijn, T., \& Komter, A. (Hrsg.). (2004). Solidarity between the sexes and the generations. Transformations in Europe. Cheltenham: Edward Elgar.

Laitinen, A., \& Pessi, A. B. (2014). Solidarity: Theory and practice. An introduction. In A. Laitinen \& A. B. Pessi (Hrsg.), Solidarity: Theory and practice (S. 1-29). Lanham et al.: Lexington Books.

Lamont, M. (2001). Symbolic boundaries: Overview. In N.J. Smelser \& P.B. Baltes (Hrsg.), International encyclopedia of the social \& behavioral sciences (S. 15341-15347). Amsterdam: Elsevier, Pergamon.

Lindenberg, S. (1998). Solidarity: Its microfoundation and macrodependence. A framing approach. In P. Doreian \& T. J. Fararo (Hrsg.), The problem of solidarity (S. 61-112). Amsterdam: Gordon and Breach.

Lindenberg, S. (2008). Social rationality, semi-modularity and goal-framing: What is it all about? Analyse \& Kritik, 30(2), 669-687.

Luhmann, N. (1984). Die Differenzierung von Interaktion und Gesellschaft. Probleme der sozialen Solidarität. In R. Kopp (Hrsg.), Solidarität in der Welt der 80er Jahre. Leistungsgesellschaft und Sozialstaat (S. 79-96). Basel: Helbing \& Lichtenhahn. 
Müller, J.-W. (2010). Verfassungspatriotismus. Frankfurt a. M.: Suhrkamp.

Ott, K. (2016). Zuwanderung und Moral. Stuttgart: Reclam.

Pettigrew, T. F. (1998). Intergroup contact theory. Annual Review of Psychology, 49(1), 65-85.

Popitz, H. (1980). Die normative Konstruktion von Gesellschaft. Tübingen: Mohr.

Prisching, M. (2003). Solidarität. Der vielschichtige Kitt gesellschaftlichen Zusammenlebens. In S. Lessenich (Hrsg.), Wohlfahrtsstaatliche Grundbegriffe. Historische und aktuelle Diskurse (S. 157-190). Frankfurt a.M.: Campus.

Rorty, R. (1992). Kontingenz, Ironie und Solidarität. Frankfurt a.M.: Suhrkamp.

Sigelman, L., \& Welch, S. (1993). The contact hypothesis revisited: Black-white interaction and positive racial attitudes. Social Forces, 71(3), 781-795.

Thome, H. (1998). Soziologie und Solidarität: Theoretische Perspektiven für die soziologische Forschung. In K. Bayertz (Hrsg.), Solidarität. Begriff und Problem (S. 217-262). Frankfurt a.M.: Suhrkamp.

Tranow, U. (2012). Das Konzept der Solidarität. Handlungstheoretische Fundierung eines soziologischen Schlüsselbegriffs. Wiesbaden: Springer VS.

Triandafyllidou, A. (1998). National identity and the "other". Ethnic and Racial Studies, 21(4), $593-612$. Werron, T. (2018). Der globale Nationalismus. Berlin: Nicolai Publishing \& Intelligence.

Wilde, L. (2007). The concept of solidarity: Emerging from the theoretical shadows. British Journal of Politics and International Relations, 9, 171-181.

Wrong, D. (1994). The problem of order: What unites and divides society? New York: Free Press.

Annette Schnabel geb. 1967. Professorin für Soziologie und Soziologische Theorien an der Heinrich-Heine-Universität in Düsseldorf. Forschungsschwerpunkte: religiöse und nationale Identitäten, Wohlfahrtsstaatsforschung, Geschlechterforschung und soziale Bewegungen. Ausgewählte aktuelle Veröffentlichungen: Sich sorgen - Eine Skizze zur Theorie der Rationalität von Sorge, in: A. Henkel, I. Karle, G. Lindemann und M. Werner (Hrsg.), Sorget nicht - Kritik der Sorge. Dimensionen der Sorge, Bd. 2, 2019; (mit M. Reddig und H. Winkel; Hrsg.) Religion im Kontext/Religion in Context - Handbuch für Wissenschaft und Studium, 2018; (mit H. Beyer) Theorien sozialer Bewegungen, 2017.

Ulf Tranow geb. 1975. Akademischer Oberrat an der Heinrich-Heine-Universität in Düsseldorf. Forschungsschwerpunkte: Solidarität und Konflikt, soziale Normen, Akteurs- und Handlungstheorie. Ausgewählte aktuelle Veröffentlichungen: Solidarity as a system of norms, in: J. Althammer (Hrsg.), Solidarity in open societies, 2019; Kontext als analytisches Konzept aus der Perspektive einer akteurszentrierten Soziologie, in: A. Schnabel, M. Reddig \& H. Winkel (Hrsg.), Religion im Kontext/Religion in Context - Handbuch für Wissenschaft und Studium, 2018; (mit T. Beckers und D. Becker) Explaining and understanding by answering "why" and "how" questions: A programmatic introduction to the special issue "Social mechanisms", in: Analyse \& Kritik, 2016. 